\title{
Number, not size, of mesenteric tumor deposits affects prognosis of small intestinal well-differentiated neuroendocrine tumors
}

\author{
Raul S. Gonzalez ${ }^{1} \cdot$ Justin M. M. Cates $10 \cdot$ Chanjuan Shi $^{2}$
}

Received: 7 February 2018 / Revised: 24 April 2018 / Accepted: 25 April 2018 / Published online: 24 May 2018

(c) United States \& Canadian Academy of Pathology 2018

\begin{abstract}
Mesenteric tumor deposits are an adverse prognostic factor for small intestinal well-differentiated neuroendocrine tumors. Per the American Joint Committee on Cancer (AJCC) Cancer Staging Manual (eighth edition), any mesenteric tumor deposit larger than $2 \mathrm{~cm}$ signifies pN2 disease. This criterion has not been critically evaluated as a prognostic factor for small intestinal neuroendocrine tumors, nor have multifocality or histologic features of mesenteric tumor deposits. We evaluated 70 small intestinal neuroendocrine tumors with mesenteric tumor deposits for lesional contour, sclerosis, inflammation, calcification, entrapped blood vessels, and perineural invasion. Ki67 proliferative indices of the largest mesenteric tumor deposit from each case were calculated, and number of tumor deposits and size of the largest deposit were recorded. Associations between these factors (along with patient age, primary tumor Ki67 index, and AJCC stage) and development of liver metastases and overall survival were assessed. Median mesenteric tumor deposit size was $1.5 \mathrm{~cm}$ (range: $0.2-7.0 \mathrm{~cm}$ ); median deposit number was 1 (range: 1-13). Primary and tumor deposit Ki67 indices within a given patient were discordant in $40 \%$ of cases but showed similar hazard ratios for disease-specific survival. Size of tumor deposits had no significant effect on prognosis, whether analyzed on a continuous scale or dichotomized using the recommended $2 \mathrm{~cm}$ cutoff. In contrast, increasing number of deposits was associated with poor prognosis, with multiple deposits conferring an 8.19-fold risk of disease-specific death compared to a single deposit $(P=0.049)$. Morphologic features of deposits had no prognostic impact. Size of mesenteric tumor deposits does not affect prognosis in small intestinal neuroendocrine tumor patients; instead, deposit multifocality is associated with shorter disease-specific survival and should be incorporated into future staging criteria.
\end{abstract}

Small intestinal well-differentiated neuroendocrine tumors are the most common primary malignancy of the small intestine [1], with an age-adjusted annual incidence of 1.0 per 100,000 for the US population for the decade 2000-2009 [2]. Their prognosis is better than for many other malignancies, with an estimated 5-year survival rate of $87 \%$ and a 10-year survival rate of $78 \%$ [2]. Still, patients can suffer significant morbidity, particularly if liver metastases give rise to carcinoid syndrome [3].

Raul S. Gonzalez

Raul_Gonzalez@URMC.Rochester.edu

1 Department of Pathology and Laboratory Medicine, University of Rochester Medical Center, Rochester, NY, USA

2 Department of Pathology, Microbiology, and Immunology, Vanderbilt University Medical Center, Nashville, TN, USA
Small intestinal neuroendocrine tumors are among several tumors that can produce mesenteric tumor deposits. They represent a form of local, rather than distant, hematogenous metastasis that is distinct from discontinuous primary tumor spread or lymph node involvement by disease [4]. Tumor deposits have been studied most extensively in colorectal carcinoma, where they have been shown to adversely affect prognosis [5]. Based on these studies, the current (eighth) edition of the American Joint Commission on Cancer (AJCC) staging system for colorectal adenocarcinoma includes classification of tumor deposits (in the absence of other nodal metastasis) as pN1c disease [6]. This criterion was included in the seventh edition as well [7].

Our research has shown that mesenteric tumor deposits associated with jejunal/ileal neuroendocrine tumors also portend worse prognosis [8] and that they appear to be a more significant predictive factor for liver metastasis than lymph node metastases [9]. Microscopically, mesenteric tumor deposits can mimic nodal disease, but they can often 
be differentiated by their irregular contours, prominent perineural invasion, and entrapped thick-walled blood vessels [8]. However, mesenteric tumor deposits may sometimes have a rounded contour and be associated with a peripheral chronic inflammatory infiltrate, thereby mimicking involved lymph nodes.

Assessment of tumor deposits was not included in the AJCC seventh edition staging for jejunal/ileal neuroendocrine tumors, but it was incorporated into the eighth edition [6]. Specifically, N-category disease is currently defined as pNO (no regional lymph node metastasis), pN1 (regional lymph node metastasis involving $<12$ nodes), and $\mathrm{pN} 2$ (large mesenteric masses [ $>2 \mathrm{~cm}]$ and/or extensive [ $\geq 12]$ nodal deposits, especially those that encase the superior mesenteric vessels). Both $\mathrm{pN} 1$ and $\mathrm{pN} 2$ categories stratify a patient as having Stage III disease. The size criterion for including mesenteric tumor deposits as $\mathrm{pN} 2$ disease has not been evaluated in the literature, although prognosis does appear worse for unresectable vs. resectable deposits [10, 11]. Therefore, we performed this study to assess whether size, number, or microscopic features of tumor deposits affects prognosis in small intestinal neuroendocrine tumors. We also studied whether immunohistochemical indices for Ki67 differ between primary tumors and their associated deposits, since we have previously observed differences in Ki67 indices between primary small intestinal neuroendocrine tumors and liver metastases [12].

\section{Materials and methods}

With Institutional Research Board approval, we searched the surgical pathology archives of Vanderbilt University Medical Center for jejunal/ileal neuroendocrine tumors with (1) hematoxylin and eosin-stained slides available for review, (2) mesenteric tumor deposits mentioned in the original pathology report or identified during prior research projects, and (3) clinical follow-up information. Mesenteric tumor deposits were defined as previously reported [4, 8]. Poorly differentiated neuroendocrine carcinomas and duodenal neuroendocrine tumors were excluded. We identified 70 cases that fulfilled all inclusion criteria; 55 of these also had prior Ki67 immunohistochemical assessment of the primary lesion(s). Thirty-two of these cases were included in a prior study on small intestinal neuroendocrine tumor mesenteric tumor deposits [8].

Slides on each case were reviewed by one pathologist (RSG), and subsequent review of all lesions interpreted as mesenteric tumor deposits was undertaken by a second pathologist (CS) for confirmation. Data recorded on each case included the World Health Organization grade [13] and AJCC T-category of the largest primary lesion and the size of the largest tumor deposit (based on gross measurements for macroscopic lesions and histologic size for microscopic lesions), as well as the number of deposits identified on histologic review. The distance of intervening non-lesional tissue between two foci of tumoral tissue required for the foci to qualify as separate discrete lesions has not been previously studied; for this project, a distance of $0.5 \mathrm{~cm}$ was used. The largest deposit from each case was also assessed for the presence or absence of perineural invasion, entrapped arteries, peripheral chronic inflammation, lesional sclerosis, and calcification, as well as whether the contour was primarily rounded or irregular. Slides marked as representing lymph nodes were also reviewed, in order to identify tumor deposits previously interpreted as nodal metastases. Patient information was compiled for each case from electronic medical records, including sex, age at time of surgery, lymph node and liver metastases, length of follow-up, and outcome at last follow-up.

Immunohistochemical stains for Ki67 were performed on the largest tumor deposit from 67 of the 70 cases. (Archived tissue was unavailable for the other three.) Four-micronthick sections from archived formalin-fixed, paraffinembedded blocks were stained using a monoclonal mouse anti-human antibody (clone MIB-1, Dako/Agilent, Santa Clara, CA; dilution 1:100). The Ki67 index for each slide was manually counted on a printed copy of a digitally captured image, as previously described [14].

Associations between clinical and pathologic factors and presence of liver metastases at presentation were assessed by logistic regression. Impact of these factors on diseasespecific survival was assessed by stratified Cox proportional hazard regression. The importance of Ki67 indices of primary small intestinal neuroendocrine tumors and their largest mesenteric tumor deposit were compared using Spearman's coefficient and post-hoc Wald linear hypothesis tests. Statistical analysis was performed using Stata v13 (Stata Corp, College Station, TX). All hypothesis tests were two-sided, with $\alpha=0.05$.

\section{Results}

Patient data is summarized in Table 1 . The study cohort consisted of 38 men and 32 women, with a median age of 60 years (range: $19-83$ years). All lesions initially deemed tumor deposits were confirmed by second review. Sixty-one (87\%) had lymph node metastases at primary resection, meaning the other nine patients $(13 \%)$ had mesenteric tumor deposits but no nodal disease. Status of liver disease was known for 69 of the 70 patients. Thirty-eight had liver metastases at initial workup, 6 developed them following surgery, and the remaining 25 had no history of liver disease at last follow-up; 43 patients with liver disease had more than one lesion at that site. All patients were AJCC 
Table 1 Clinicopathologic features of small intestinal welldifferentiated neuroendocrine tumor patients with mesenteric tumor deposits

\begin{tabular}{|c|c|}
\hline Age (median; range) & 60 years; $19-83$ years \\
\hline Sex & 38 male:32 female \\
\hline $\begin{array}{l}\text { Number of mesenteric } \\
\text { tumor deposits (median; } \\
\text { range) }\end{array}$ & $\begin{array}{l}1 ; 1-13 \text { (innumerable in } \\
\text { two patients) }\end{array}$ \\
\hline $\begin{array}{l}\text { Size of largest mesenteric } \\
\text { tumor deposit (median; } \\
\text { range) }\end{array}$ & $1.5 \mathrm{~cm} ; 0.2-7.0 \mathrm{~cm}$ \\
\hline $\begin{array}{l}\text { Mesenteric tumor deposit } \\
\text { perineural invasion }\end{array}$ & $59(84 \%)$ \\
\hline $\begin{array}{l}\text { Mesenteric tumor deposit } \\
\text { irregular contour }\end{array}$ & $57(81 \%)$ \\
\hline $\begin{array}{l}\text { Mesenteric tumor deposit } \\
\text { peripheral inflammation }\end{array}$ & $28(40 \%)$ \\
\hline $\begin{array}{l}\text { Mesenteric tumor deposit } \\
\text { calcification }\end{array}$ & $12(17 \%)$ \\
\hline $\begin{array}{l}\text { Mesenteric tumor deposit } \\
\text { entrapped vessels }\end{array}$ & $57(81 \%)$ \\
\hline $\begin{array}{l}\text { Mesenteric tumor deposit } \\
\text { sclerosis }\end{array}$ & $47(67 \%)$ \\
\hline Lymph node metastasis & $61(87 \%)$ \\
\hline $\begin{array}{l}\text { Liver metastasis at last } \\
\text { follow-up }\end{array}$ & $44 / 69(64 \%)$ \\
\hline \multicolumn{2}{|c|}{$\begin{array}{l}\text { American Joint Committee on Cancer eighth edition combined } \\
\text { pathologic stage }\end{array}$} \\
\hline I & $0(0 \%)$ \\
\hline II & $1(1 \%)$ \\
\hline III & $30(43 \%)$ \\
\hline IV & $39(56 \%)$ \\
\hline $\begin{array}{l}\text { Follow-up length (median; } \\
\text { range) }\end{array}$ & 52 months; $1-190$ months \\
\hline \multicolumn{2}{|l|}{ Outcome at last follow-up } \\
\hline $\begin{array}{l}\text { Alive, no evidence of } \\
\text { disease }\end{array}$ & $19(27 \%)$ \\
\hline Alive with disease & $29(41 \%)$ \\
\hline Died of disease & $19(27 \%)$ \\
\hline $\begin{array}{l}\text { Died of other/unknown } \\
\text { cause }\end{array}$ & $3(4 \%)$ \\
\hline
\end{tabular}

stage III or IV at diagnosis, except for one who was stage II (no nodal disease, one $0.2 \mathrm{~cm}$ deposit). No patients had a tumor deposit larger than $2 \mathrm{~cm}$ without also having nodal or distant metastases; in other words, no patients met AJCC eighth edition $\mathrm{pN} 2$ criteria without also meeting $\mathrm{pN} 1$ or pM1 criteria.

The median size of tumor deposits was $1.5 \mathrm{~cm}$ (range, $0.2-7.0 \mathrm{~cm}$ ), and the median number of deposits was 1 (range, 1-13). For 64 cases, all original slides were available for review. Among these cases, 39 (61\%) had only a single deposit (Fig. 1a), 23 (36\%) had between 2 and 13 deposits, and 2 (3\%) had innumerable tiny deposits studding the mesentery (arguably interpretable as "carcinomatosis," but recorded as tumor deposits for the purpose of this study, given their anatomic location) (Fig. 1b). Only pathologic details of the largest tumor deposit were recorded for the remaining six cases lacking some of the original H\&E slides; total tumor deposit count was not included due to incomplete information.

Review of the largest mesenteric tumor deposit in each case showed that $59(84 \%)$ were associated with perineural invasion (Fig. 1c), 57 (81\%) contained entrapped blood vessels (Fig. 1c), 28 (40\%) had peripheral inflammation (Fig. 1d,e), 47 (67\%) were sclerotic, 12 (17\%) harbored dystrophic calcifications (Fig. 1f), and 13 (19\%) demonstrated a rounded contour (Fig. 1f) for more than half of its circumference, instead of an entirely irregular border (see Fig. 1a).

Most mesenteric tumor deposits $(54,77 \%)$ were grade 1 based on Ki67 proliferation index and mitotic rate; the remaining 16 (23\%) were grade 2 . Of the 55 primary small intestinal neuroendocrine tumors with Ki67 data, 42 were grade 1; the largest tumor deposits from this group were predominantly grade 1 (39 tumors) but occasionally grade 2 (3 tumors). Similarly, among the 13 grade 2 primary tumors, the largest deposit was grade 1 in 12 cases and grade 2 in 1 case $(P=0.65)$. Overall, Ki67 indices of primary tumors and their associated deposits were not strongly correlated (Spearman's rank coefficient, $0.23 ; P=0.13$ ). The hazard ratio for disease-specific survival for the Ki67 indices of tumor deposits appeared statistically significant $(P=0.024)$, unlike the Ki67 indices for primary lesions $(P$ $=0.76$ ); however, these coefficients were not significantly different from each other in post-hoc Wald linear hypothesis tests $(P=0.30)$.

After controlling for covariates, only one factor was significantly associated with the presence of liver disease at presentation: An irregular contour for the largest deposit signified a higher risk of hepatic disease (odds ratio 4.65; 95\% CI 1.01-21.28; $P=0.048)$. Neither deposit size $(P=$ $0.68)$ nor increasing deposit number $(P=0.091)$ increased the risk of liver metastasis.

Results of univariate survival analysis, stratified by patient age and by AJCC pT, pN, and pM status, are shown in Table 2. Size of mesenteric tumor deposit was not statistically significant when analyzed either as a binary variable using the $2 \mathrm{~cm}$ cutoff recommended by the AJCC $(P=$ $0.36)$ (Fig. 2a) or as a continuous variable $(P=0.89)$. In contrast, increasing number of tumor deposits was associated with increased risk of disease-specific death when analyzed either as a continuous variable (HR 1.52, $P=$ $0.024)$ and when dichotomized as single vs. multiple deposits (HR 8.19, $P=0.049$ ) (Fig. 2b).

A multivariate survival analysis including number of tumor deposits as a factor is shown in Table 3. Deposit 

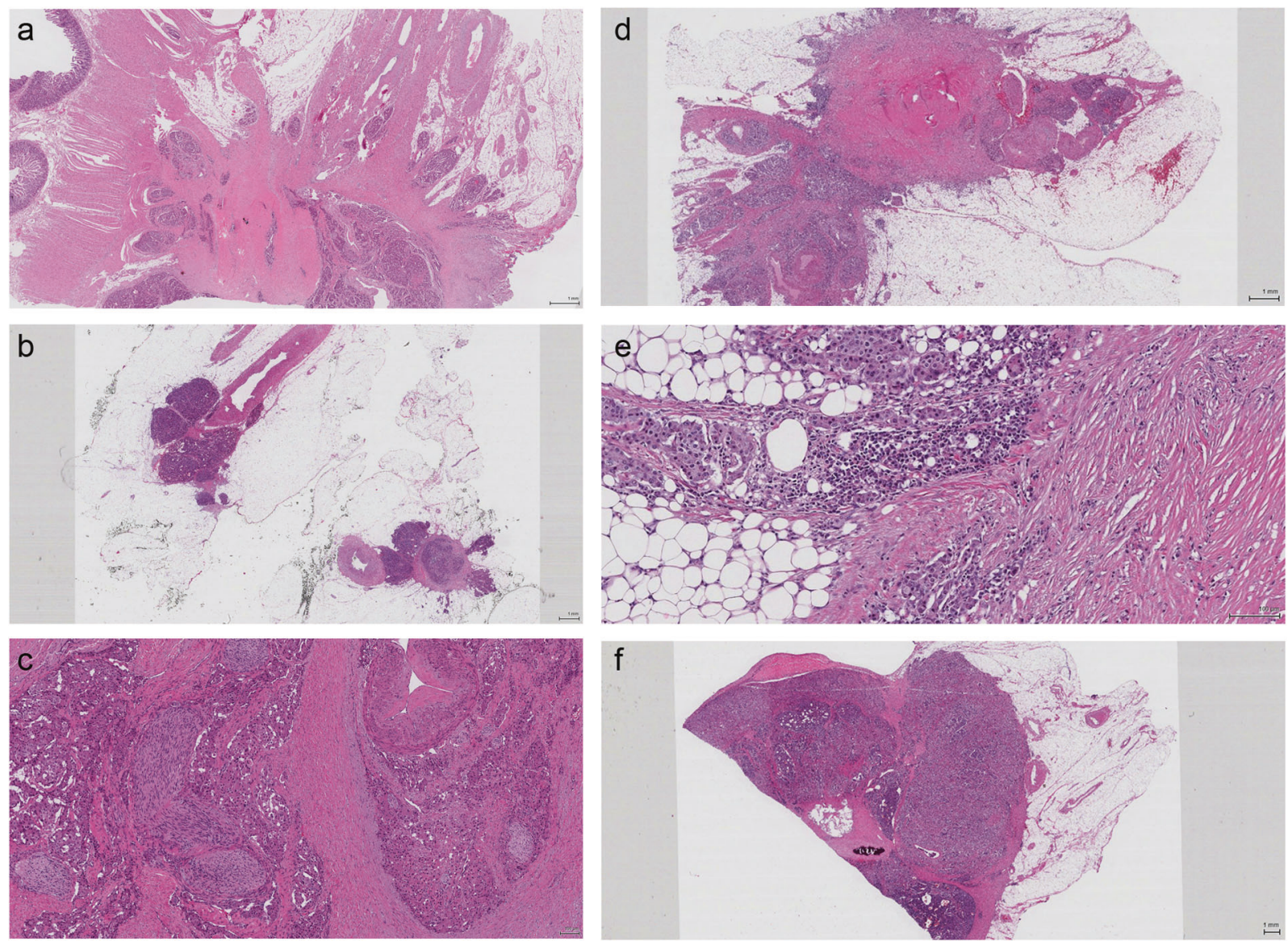

Fig. 1 Representative microscopic features of small intestinal neuroendocrine tumor mesenteric tumor deposits. a A large, solitary tumor deposit with a typical irregular contour. b Multiple small deposits associated with blood vessels and studding the mesentery. c A tumor

deposit with characteristic perineural invasion and entrapped blood vessels. d, e Peripheral inflammation around a tumor deposit. f A tumor deposit with a rounded, rather than irregular, contour. Dystrophic calcification is also present

number remained statistically significant $(P=0.007)$, whereas deposit size was not germane to the final model. Lymph node metastases did not significantly impact patient survival in this analysis. Single vs. multiple deposits was not included in this analysis due to overlapping relevance; in a separate multivariate survival analysis incorporating this factor rather than deposit number (not shown), multiple deposits was also found prognostically relevant $(P=$ 0.046).

\section{Discussion}

Mesenteric tumor deposits, which are distinct from lymph nodes completely effaced by metastatic tumor, have been recognized as a potential component of tumor staging since the AJCC fifth edition [5], where colorectal carcinomas with tumor deposits were upstaged in either T-category or $\mathrm{N}$-category disease, depending on the size of the deposit. The AJCC seventh edition introduced the concept of $\mathrm{pN} 1 \mathrm{c}$, which assigned specific importance to colorectal tumor deposits in the absence of nodal disease. Although tumor deposits have been identified in other gastrointestinal malignancies, including gastric and pancreatic adenocarcinoma [15], they were not incorporated into staging algorithms for other sites until the AJCC eighth edition, where deposits $>2 \mathrm{~cm}$ are now considered $\mathrm{pN} 2$ disease for jejunal/ ileal neuroendocrine tumors. While this size criterion is unsupported by currently published data, the AJCC did note that the new pN2 category was included solely for data collection in order to evaluate prospectively its clinical utility and that it purposefully does not alter overall patient stage compared to $\mathrm{pN} 1$ disease [6].

In our study of 70 small intestinal neuroendocrine tumor patients with mesenteric tumor deposits, there was no evidence that deposit size affected disease-specific survival, either as a continuous variable or dichotomized at the recommended $2 \mathrm{~cm}$ cutoff. In contrast, increasing number of deposits was associated with decreased disease-specific survival. Patients with multiple deposits also showed a much higher hazard ratio compared to patients with a single deposit. Although the study design did not include patients 
Table 2 Univariate survival analysis for small intestinal welldifferentiated neuroendocrine tumor patients with mesenteric tumor deposits, stratified by age and American Joint Committee on Cancer pT, pN, and pM status

\begin{tabular}{llll}
\hline Variable & Hazard ratio & $95 \%$ confidence interval & $P$-value \\
\hline Male sex & 0.73 & $0.21-2.49$ & 0.61 \\
Primary lesion Ki67 (1\% increments) & 1.05 & $0.77-1.44$ & 0.76 \\
Mesenteric tumor deposit Ki67 (1\% increments) & 1.52 & $1.06-2.19$ & 0.024 \\
$>$ 1 Mesenteric tumor deposit (vs. 1 deposit) & 8.19 & $1.01-66.43$ & 0.049 \\
Number of mesenteric tumor deposits (1-deposit & 2.50 & $1.10-5.69$ & 0.029 \\
increments) & & & \\
Mesenteric tumor deposit size (1 cm increments) & 0.89 & $0.52-1.52$ & 0.68 \\
Mesenteric tumor deposit size (>2 cm vs. $\leq 2 \mathrm{~cm})$ & 0.36 & $0.06-2.17$ & 0.27 \\
Mesenteric tumor deposit perineural invasion & 0.36 & $0.05-2.67$ & 0.32 \\
Mesenteric tumor deposit irregular contour & 1.53 & $0.17-14.08$ & 0.71 \\
Mesenteric tumor deposit peripheral inflammation & 2.20 & $0.62-7.88$ & 0.23 \\
Mesenteric tumor deposit calcification & 1.19 & $0.21-6.62$ & 0.85 \\
Mesenteric tumor deposit entrapped vessels & 1.40 & $0.25-7.82$ & 0.70 \\
Mesenteric tumor deposit sclerosis & 0.47 & $0.12-1.87$ & 0.29
\end{tabular}
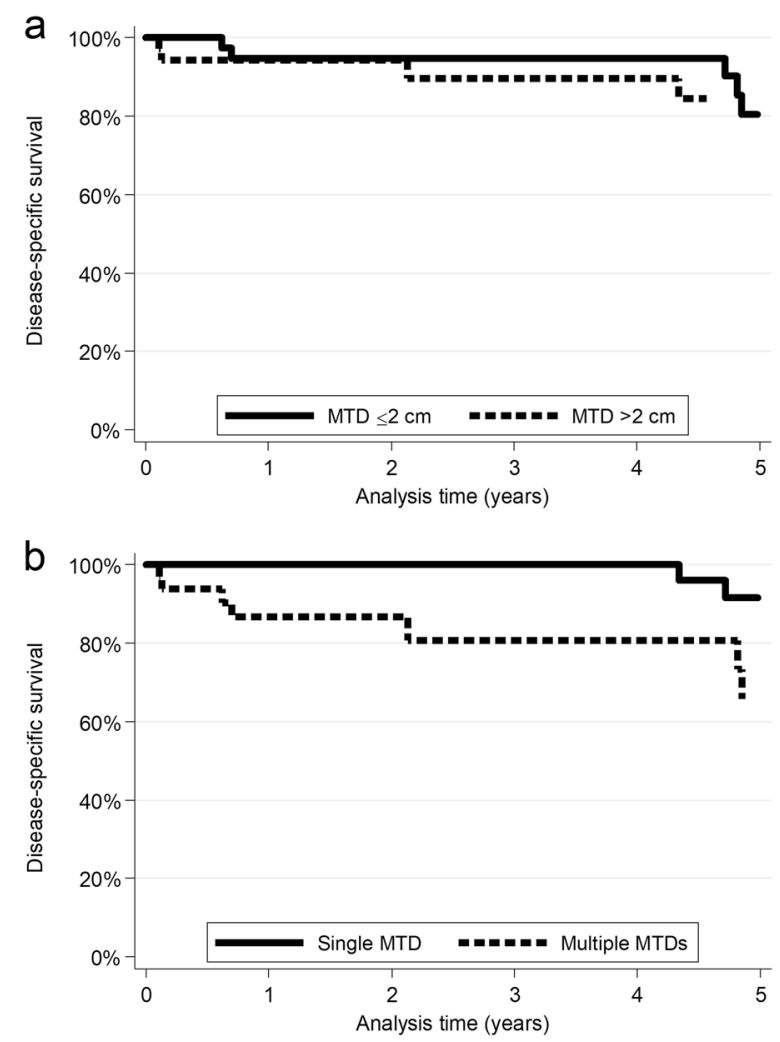

Fig. 2 Kaplan-Meier age- and American Joint Committee on Cancer stage-adjusted disease-specific survival curves for small intestinal neuroendocrine tumor patients. a Comparison of patients with mesenteric tumor deposits $\leq 2$ and $>2 \mathrm{~cm}$, showing no statistically significant difference in survival. b Comparison of patients with one tumor deposit and multiple deposits, showing a statistically significant difference in survival $(P=0.049)$

without deposits, we have previously shown that the presence of any number of deposits portends a worse prognosis in small intestinal neuroendocrine tumors [8]. Therefore, the AJCC eighth edition $\mathrm{pN} 2$ criterion for tumor deposits
Table 3 Multivariate survival analysis for small intestinal welldifferentiated neuroendocrine tumor patients with mesenteric tumor deposits

\begin{tabular}{llll}
\hline Variable & $\begin{array}{l}\text { Hazard } \\
\text { ratio }\end{array}$ & $\begin{array}{l}\text { 95\% confidence } \\
\text { interval }\end{array}$ & $P$-value \\
\hline Age & 1.06 & $1.00-1.13$ & 0.052 \\
Lymph node metastasis & 2.06 & $0.29-14.54$ & 0.47 \\
$\begin{array}{l}\text { Number of mesenteric } \\
\text { tumor deposits (1-deposit }\end{array}$ & 1.35 & $1.09-1.67$ & 0.007 \\
\begin{tabular}{l} 
increments) \\
\hline
\end{tabular} & & & \\
\hline
\end{tabular}

appears to have no significant prognostic impact. Moreover, as currently evaluated, deposits $\leq 2 \mathrm{~cm}$ are ignored in the staging algorithm.

Distinguishing mesenteric tumor deposits from effaced lymph nodes is challenging, as previous studies have asserted [4]. However, the presence of entrapped nerves and thick-walled arteries within an extramural discontinuous metastatic focus supports interpretation as a tumor deposit, as these anatomic structures are uncommon within mesenteric lymph nodes. Similarly, an irregular contour suggests haphazard growth of a soft tissue deposit of neuroendocrine tumor, in contrast to tumor confined by a lymph node capsule. Nevertheless, lymph node metastases can develop an irregular contour once extranodal extension has occurred. Another potential pitfall is the presence of a peripheral rim of chronic inflammation surrounding a tumor deposit. If simply composed of scattered inflammatory cells, the lesion can be presumed to be a deposit, but if the inflammatory infiltrate is organized into lymphoid follicles with germinal centers, care should be taken to search for subcapsular sinuses or other features that would identify the inflammation as residual lymph node tissue. Each of these morphologic criteria was applied in this study, with the final determination relying on overall impression. The 
interobserver variability in distinguishing tumor deposits from effaced lymph nodes appears moderate in colorectal carcinoma, with a reported $\kappa$ statistic of 0.48 among gastrointestinal pathologists [4]. To ensure consistency, only one pathologist reviewed the cases in the present study, using the criteria and experience gained from our previous studies on mesenteric tumor deposits [8, 9]. A second pathologist re-reviewed and confirmed the diagnosis of mesenteric tumor deposits in all cases; however, given our group's familiarity and experience with these lesions based on our previous work, the true interobserver variability for diagnosis of neuroendocrine tumor deposits remains unclear, especially for pathologists less familiar with them.

We also compared the Ki67 staining index of primary lesions and their largest associated tumor deposit. Previously, we have shown that small intestinal neuroendocrine tumors with low Ki67 proliferative indices may give rise to hepatic metastases with higher indices, sometimes increasing WHO grade [12]. In that prior study, larger metastatic nodules in the liver were generally associated with higher Ki67 indices, so we chose to perform immunohistochemistry on the largest tumor deposit from each patient in the current study. Here, we found no evidence of correlation between the Ki67 proliferation indices of the primary tumor and its largest associated deposit, with differences in WHO grade apparent in more than $40 \%$ of cases (including both upgrading and downgrading). Although hazard ratios for tumor deposit Ki67 indices were greater than those of primary small intestinal neuroendocrine tumors, more data points were available for the former, and the difference was not statistically significant. Therefore, we cannot make definitive conclusions regarding which tumor site is optimal for Ki67 immunohistochemistry based on our results. Regardless, Ki67 staining of subsequently resected metastatic or recurrent lesions (as is the case for most liver metastases) remains advisable to reassess the current grade of the patient's disease [12].

The new AJCC pN2 criteria for staging jejunal/ileal neuroendocrine tumors include cases with $\geq 12$ positive lymph nodes. While this specific numerical cutoff has not been evaluated, a higher ratio of positive to total lymph nodes adversely affects patient prognosis in small intestinal neuroendocrine tumors [16]. Additionally, in describing pN2 criteria, the AJCC emphasizes mesenteric tumor deposits or nodal disease that "encase[s] the superior mesenteric vessels." This likely refers to the morbidity and mortality associated with tumor-induced fibrosis around the mesenteric vessels [17]. While many tumor deposits in our study involved mesenteric vessels, we did not evaluate this aspect of the new N-category; to our knowledge, the specific impact of metastatic disease involving superior mesenteric vessels has not been assessed in rigorous multivariable analysis.
The recently adopted AJCC eighth edition N-category criteria for small intestinal neuroendocrine tumors include multiple parameters specifically designated for future scrutiny. This study determined that the new size criterion $(2$ $\mathrm{cm}$ ) for mesenteric tumor deposits has no prognostic significance, whereas multiple deposits do adversely impact prognosis. Future iterations of the small intestinal neuroendocrine tumor staging system should therefore omit this arbitrary size requirement and consider multifocality of tumor deposits as a more clinically relevant staging criterion. The number of involved lymph nodes and specific anatomic location of metastatic tumor deposits require further study.

\section{Compliance with ethical standards}

Conflict of interest The authors declare that they have no conflict of interest.

\section{References}

1. Bilimoria KY, Bentrem DJ, Wayne JD, Ko CY, Bennett CL, Talamonti MS. Small bowel cancer in the United States: changes in epidemiology, treatment, and survival over the last 20 years. Ann Surg. 2009;249:63-71.

2. Mocellin S, Nitti D. Gastrointestinal carcinoid: epidemiological and survival evidence from a large population-based study $(\mathrm{n}=25$ 531). Ann Oncol. 2013;24:3040-4.

3. Modlin IM, Kidd M, Latich I, Zikusoka MN, Shapiro MD. Current status of gastrointestinal carcinoids. Gastroenterology. 2005; 128:1717-51.

4. Rock JB, Washington MK, Adsay NV, et al. Debating deposits: an interobserver variability study of lymph nodes and pericolonic tumor deposits in colonic adenocarcinoma. Arch Pathol Lab Med. 2014;138:636-42.

5. Jin M, Roth R, Rock JB, Washington MK, Lehman A, Frankel WL. The impact of tumor deposits on colonic adenocarcinoma AJCC TNM staging and outcome. Am J Surg Pathol. 2015;39:109-15.

6. Amin MB, et al. AJCC Cancer Staging Manual. 8th ed. New York, NY: Springer; 2017. p. 375-87.

7. Edge SB, Fritz AG, Byrd DR, et al. AJCC Cancer Staging Manual. 7th ed. New York, NY: Springer; 2009. p. 181-5.

8. Gonzalez RS, Liu EH, Alvarez JR, Ayers GD, Washington MK, Shi C. Should mesenteric tumor deposits be included in staging of well-differentiated small intestine neuroendocrine tumors? Mod Pathol. 2014;27:1288-95.

9. Fata CR, Gonzalez RS, Liu E, Cates JM, Shi C. Mesenteric tumor deposits in midgut small intestinal neuroendocrine tumors are a stronger indicator than lymph node metastasis for liver metastasis and poor prognosis. Am J Surg Pathol. 2017;41: 128-33.

10. Hellman P, Lundström T, Ohrvall $U$, et al. Effect of surgery on the outcome of midgut carcinoid disease with lymph node and liver metastases. World J Surg. 2002;26:991-7.

11. Strosberg JR, Weber JM, Feldman M, Coppola D, Meredith K, Kvols LK. Prognostic validity of the American Joint Committee on Cancer staging classification for midgut neuroendocrine tumors. J Clin Oncol. 2013;31:420-5. 
12. Shi C, Gonzalez RS, Zhao Z, et al. Liver metastases of small intestine neuroendocrine tumors: Ki-67 heterogeneity and World Health Organization grade discordance with primary tumors. Am J Clin Pathol. 2015;143:398-404.

13. Rindi G, Arnold R, Bosman FT, et al.. Nomenclature and classification of neuroendocrine neoplasms of the digestive system. In: Bosman FT, Carneiro F, Hruban RH, Theise ND, editors. WHO classification of tumors of the digestive system. 4th ed. Lyon: IARC; 2010. p. 13-14.

14. Reid MD, Bagci P, Ohike N, et al. Calculation of the Ki67 index in pancreatic neuroendocrine tumors: a comparative analysis of four counting methodologies. Mod Pathol. 2015;28:686-94.
15. Puppa G, Ueno H, Kayahara M, et al. Tumor deposits are encountered in advanced colorectal cancer and other adenocarcinomas: an expanded classification with implications for colorectal cancer staging system including a unifying concept of intransit metastases. Mod Pathol. 2009;22:410-5.

16. Kim MK, Warner RR, Ward SC, et al. Prognostic significance of lymph node metastases in small intestinal neuroendocrine tumors. Neuroendocrinology. 2015;101:58-65.

17. Landau M, Wisniewski S, Davison J. Jejunoileal neuroendocrine tumors complicated by intestinal ischemic necrosis are associated with worse overall survival. Arch Pathol Lab Med. 2016;140:461-6. 